\title{
How equitable is access to treatment for lung cancer patients? A population-based review of treatment practices in Ontario
}

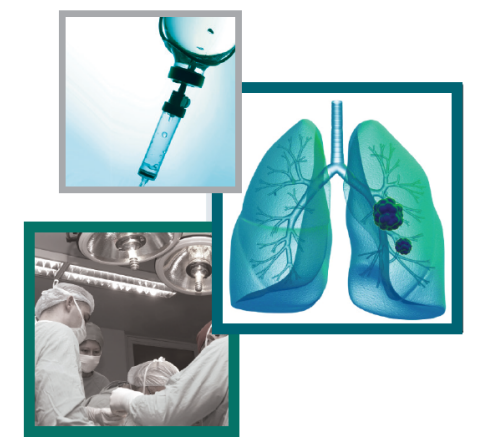

\author{
William K Evans*,1, Jennifer Stiff², Kelly J Woltman ${ }^{3}$, Yee C Ung ${ }^{4}$, Sue Su-Myat ${ }^{5}$, Phongsack \\ Manivong ${ }^{3}$, Kyle Tsang ${ }^{3}$, Narges Nazen-Rad ${ }^{3}$, Aryn Gatto ${ }^{5}$, Ashley Tyrrell ${ }^{5}$, Rebecca Anas ${ }^{2}$, \\ Gail Darling ${ }^{5}$ \& Carol Sawka \\ ${ }^{1}$ McMaster University, Department of Oncology, Hamilton, Ontario, Canada \\ ${ }^{2}$ Cancer Quality Council of Ontario Secretariat, Clinical Programs \& Quality Initiatives, Cancer Care Ontario, Toronto, Ontario, \\ Canada \\ ${ }^{3}$ Cancer Analytics, Cancer Care Ontario, Toronto, Ontario, Canada \\ ${ }^{4}$ Odette Cancer Center, University of Toronto, Toronto, Ontario, Canada \\ ${ }^{5}$ Disease Pathway Management, Clinical Programs \& Quality Initiatives, Cancer Care Ontario, Toronto, Ontario, Canada \\ ${ }^{6}$ Cancer Care Ontario, University of Toronto, Toronto, Ontario, Canada \\ * Author for correspondence: Tel.: +1 905527 6497; Fax: +1 905527 6497; bill.evans@cancercare.on.ca
}

\section{Practice points}

- In a population-based study, lung cancer surgical resection rates in patients with early stage NSCLC were only modestly greater than $50 \%$.

- Resection rates vary by disease stage, patient age, income level and immigrant status.

- Patients 80 years and older infrequently $(26.8 \%)$ undergo surgical resection.

- Resection rates and adjuvant chemotherapy (AC) administration rates were significantly higher in the highest income regions compared with the lowest despite access to universal healthcare.

- Just over $50 \%$ of patients who are candidates for $A C$ receive it but rates vary by health region.

- Patients 65 years or older were significantly less likely to receive AC compared with younger patients.

Aim:Guideline concordance is one of the metrics used by the Cancer Quality Council of Ontario and Cancer Care Ontario to assess the quality of cancer care and to drive quality improvement. Materials \& Methods: The rates for lung cancer surgical resection and concordance with the Cancer Care Ontario postoperative adjuvant chemotherapy (AC) guideline were assessed by health region during two time periods (20102011 and 2012-2013) according to five equity measures (age, sex, neighborhood income, location of residence and size of immigrant population). Results: Of the patients with stage I/II NSCLC, $52.2 \%$ to $63.0 \%$ underwent surgical resection in the province of Ontario, Canada; for patients with stage IIIA disease, the rate was $26.4 \%$. The probability of a surgical resection decreased substantially with age; only $26.9 \%$ of those with potentially resectable (stage I-IIIA) disease over 80 years underwent surgery. The use of postoperative AC increased modestly over the time of the study but the rate of use varied widely by health region (34.6 to $84.6 \%$ ). Patients in rural areas were as likely to receive AC as urban dwellers; however, older aged patients ( $\geq 65$ years) and those from the lowest income neighborhoods were significantly less likely to receive AC. Conclusion: Surgical rates and the use of AC vary by health region in Ontario and by age and level of neighborhood income despite universal access in a publicly funded health care system. The reasons for this variance are unclear but warrant further study.

Presented in part at the 15th World Conference on Lung Cancer, Sydney, Australia, 27-30 October 2013

First draft submitted: 6 July 2017; Accepted for publication: 27 September 2017; Published online:

1 December 2017

Keywords: access to treatment $\bullet$ equity $\bullet$ lung cancer

Cancer Care Ontario (CCO) is the provincial agency that drives continuous quality improvement in cancer prevention, screening and care delivery in Ontario, Canada [1]. Through its Program in Evidence-based Care, 
CCO has developed practice guidelines for the care of cancer patients in partnership with clinical experts [2]. The provincial Lung Cancer Disease Site Group develops guidelines for thoracic cancers and these guidelines are posted on CCO's website [3].

The Cancer Quality Council of Ontario advises CCO on quality issues and in 2008 began to publicly report on the degree of concordance of selected practice guidelines as part of a set of performance metrics that make up the Cancer System Quality Index (CSQI) [4,5]. The CSQI is a web-based report of quality indicators that span the cancer care continuum according to the seven dimensions of quality [6]. Concordance of practice with guideline recommendations is considered both a measure of effective care and equity.

One of the guidelines selected for review and public reporting was the guideline "Postoperative adjuvant chemotherapy $(A C)$ with or without radiotherapy in completely resected NSCLC", which was jointly published with the American Society of Oncology in 2007 [7] with updates in 2012 and 2016 [8,9].The guideline recommends postoperative adjuvant cisplatin-based chemotherapy (AC) in adult patients who have undergone complete resection of stage II and stage IIIA NSCLC based on the National Cancer Institute of Canada's NCIC BR 10 trial [10], other adjuvant trials [11-16] and two meta-analyses [17,18].

Although progress in the treatment of NSCLC has been slow, the survival of surgically resected NSCLC patients has improved with postoperative AC. The BR 10 trial demonstrated a $15 \%$ increase in absolute survival at 5 years [10] and this benefit largely persisted at 10 years [19]. Adjuvant vinorelbine-cisplatin has a low toxicity profile and is cost-effective [20]. Therefore, it was expected that AC would be rapidly adopted by oncologists and used in the majority of surgically resected stage II/IIIA NSCLC patients.

Between 2001 and 2006, there was a sharp uptake of this practice in Ontario, with the frequency of administration of adjuvant chemotherapy rising from 7 to $31 \%$ by 2006 [21]. It was assumed that this upward trend would continue given the results of the clinical trials.

This study evaluates the degree of concordance between the AC guideline and actual practice at the population level. It also assesses the relationship between various equity measures and guideline concordance during two sequential 2-year time periods - January 2010 to December 2011 (Cohort A) and January 2012 to December 2013 (Cohort B). The frequency of surgical resection in patients with potentially resectable NSCLC in Ontario is also assessed to better understand the population for whom AC was appropriate.

\section{Methods}

Patients diagnosed between 2010 and 2013 with lung cancer were identified from the Ontario Cancer Registry (OCR) using the lung topology ICD-O-3 code C 34 . The OCR is a patient-specific population-based cancer registry, which captures all newly diagnosed cases in the province of Ontario. Patients were excluded from the study cohorts according to the flow chart shown in Figure 1 beginning with exclusions if they did not have a valid Health Insurance Number (HIN), had unknown or nondiagnostic morphology, were not residents of Ontario, or had a missing postal code. From this initial cohort, small cell lung cancer patients were identified and excluded using a list of morphology codes (Appendix Table A).

To determine surgical resection rates, all NSCLC cases defined by ICD-O-3 codes and identified in the Ontario Cancer Registry were linked to the Canadian Institute for Health Information (CIHI) Discharge Abstract Database (DAD) for in-patient surgical activity and to National Ambulatory Care Reporting System (NACRS) for outpatient surgical activity. A list of Canadian Classification of Health Intervention lung codes were used (Appendix Table B) to identify relevant surgical procedures.

Pathology was coded according to Canadian Cancer Registry requirements using the WHO's International Classification of Diseases for Oncology, 3rd Edition [22]. Data on the method of tissue acquisition or the use of immunohistochemical staining were not collected.

Best available stage was obtained from standardized synoptic pathology reports using the Collaborative Staging System [23]. The Collaborative Staging data collection system utilizes a computer algorithm to extract data elements on tumor characteristics (tumor size, nodal involvement, presence of metastases), abstracted by the cancer registry analyst from different data bases, including hospital electronic patient records, the Activity Level Reporting system at CCO, a database that contains pathology reports submitted from laboratories, the DAD and NACRS data. The DAD is a national database that contains information on separations from acute-care institutions, including discharges, deaths, sign-outs and transfers. NACRS is a national database that collects demographic, administrative, clinical and service-specific data for emergency department visits, day surgery and other ambulatory care visits. From these data holdings, the tumor characteristics and method of their determination (clinical versus pathological) 


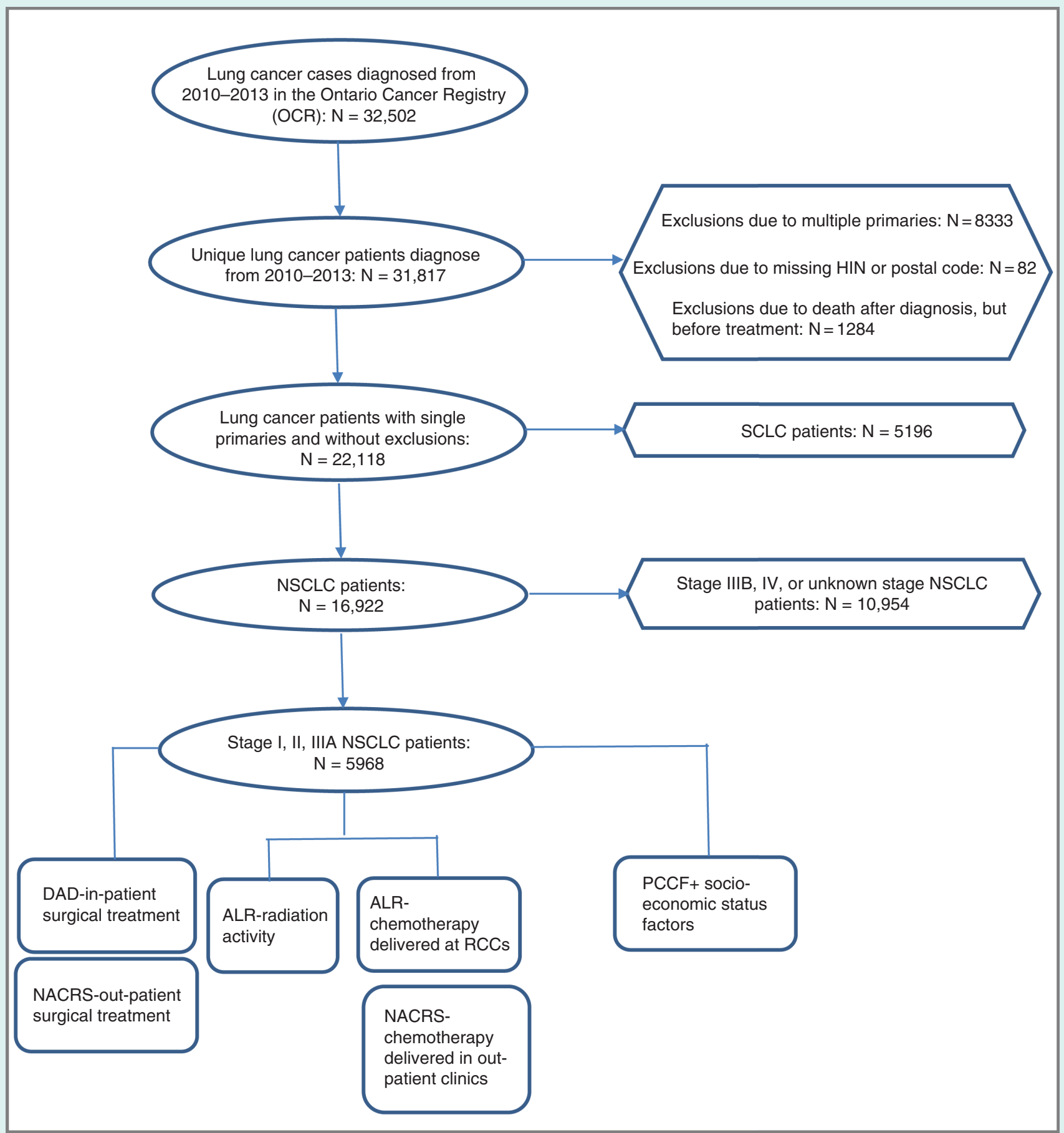

Figure 1. Flow chart showing how the non-small-cell lung cancer cohorts were constructed.

ALR: Activity level reporting; DAD: Discharge abstract database; NACRS: National ambulatory care reporting system; PCCF: Postal code conversion file.

are evaluated using a decision hierarchy to derive the collaborative stage. Collaborative stage integration software then links the stage group to the corresponding case in the OCR. Where there is a match, the stage group then populates the 'Best Stage Table.' Internal inter-rater reliability audits conducted between 2010 and 2015 have consistently shown an agreement rate of $90 \%$ or higher for all lung cancer data elements. 
Two cohorts of NSCLC patients undergoing surgical resection of stage II and IIIA NSCLC were assessed for postoperative therapy. Cohort A consisted of patients resected within 270 days of diagnosis between January 2010 and December 2011; patients in Cohort B were resected within the same time interval from diagnosis between January 2012 and December 2013. Patient data from each of the cohorts were linked to CCO's Activity Level Reporting database, which contains regimen-specific systemic treatment information from the Regional Cancer Centres (RCCs) across Ontario. Data on whether chemotherapy was delivered in non-RCC facilities were obtained through record linkage to the DAD and NACRS; however, information on the specific type of chemotherapy is not available through NACRS. Postoperative treatments were grouped as follows: platinum-based (cisplatin or carboplatin) AC given within 120 days of surgical resection administered in an RCC; any chemotherapy administered in a non-RCC treatment facility within 120 days of surgery on the assumption that chemotherapy administered postoperatively in this time frame was $\mathrm{AC}$ and guideline concordant; other non-platinum-based chemotherapy within 120 days; and no treatment. The time intervals used to define the cohorts are arbitrary but were recommended by disease site experts when the guideline concordance metrics were established for the CSQI. Non-RCC facilities were included in this analysis in order to have as complete a picture of the population-based treatments as possible.

Guideline concordance was examined for each of the two cohorts by health region and by the following measures of equity: age (30-49, 50-64, 65-79, 80+ years), sex (male, female), neighborhood income (reported in quintiles), location of residence (urban, rural, rural-remote, rural very remote as defined by Statistics Canada) [24] and size of immigrant population (reported in terciles). For the purpose of the study, the definition of an immigrant is a person who is or has ever been a landed immigrant and granted the right to live in Canada permanently by immigration authorities.

Surgical and AC administration rates were assessed in relation to equity measures by geographic region by linking the postal codes of patients to their corresponding geographic area using the Postal Code Conversion File Plus managed by Statistics Canada. Information on neighborhood income (reported in quintiles with quintile 1 having the lowest income and quintile 5 the highest), location of residence (urban, rural, rural-remote, rural very remote) [24] and size of immigrant population (reported in terciles with tercile 1 having the lowest immigrants and tercile 3 the highest immigrant population) was derived from the Postal Code Conversion File Plus.

Data runs were completed for guideline concordance in July 2015 and for surgical management in July 2016.

Multiple Chi-Square tests of homogeneity between various factors including age, gender, socioeconomic factors and location of residence were used to examine resection rates among NSCLC patients. Additionally, pairwise comparisons of the rates of AC between Cohort A and Cohort B for each local health integration network (LHIN) were performed using a Chi-square test of homogeneity each with one degree of freedom. As the number of resected cases in each LHIN and cohort were small, caution needs to be exercised in interpreting any significance result at the LHIN level. Chi-square tests were run in SAS Version 9 using the proc freq procedure. Logistic regression analysis was not undertaken.

\section{Results}

From 1 January 2010 to 31 December 2013, 32,502 cases of lung cancer were diagnosed in Ontario. There were 22,118 patients with a single primary. 8333 patients had multiple primaries and were excluded from the analysis. The percentage of stage I-III NSCLC cases with multiple primary cancers was approximately $23 \%$ and varied by stage and year of diagnosis. The most common second primaries were lung (14.4\%), colorectal cancer (11.9\%), breast cancer (10.6\%) and prostate cancer (6.3\%). A total of 82 patients were excluded for missing health information numbers or postal codes; 1264 patients died before any treatment was given.

Of the patients with a single primary, 16,922 (76.5\%) had non-small-cell histology: 8448 patients were in cohort A and 8474 patients in cohort B.

Overall, $89.8 \%$ of all NSCLC patients $(15,200)$ were staged (Cohort $93.5 \%$; Cohort B $86.1 \%$ ) and of these, 5968 patients were staged as stage I, II, or IIIA and were, therefore, potentially resectable: 2885 in Cohort A and 3083 in Cohort B. Of these, 2787 (46.7\%) actually underwent resection: 52.2 to $63.0 \%$ of patients with stage I or II disease and $26.4 \%$ with stage IIIA disease. Resection rates decreased with age: $54.7 \%$ for ages $50-64,47.4 \%$ for ages $65-79$ and $26.9 \%$ for those 80 and older (Table 1 ). 
Table 1. Descriptive statistics for non-small-cell lung cancer patients (stages I, II, IIIA) who underwent surgical resection versus those who did not.

\begin{tabular}{|c|c|c|}
\hline & No. resected (\%) & No. not resected (\%) \\
\hline \multicolumn{3}{|l|}{ Age group } \\
\hline$-19-49$ & 109 (61.6) & $68(38.4)$ \\
\hline$-50-64$ & $933(54.7)$ & $773(45.3)$ \\
\hline$-65-79$ & $1495(47.4)$ & $1662(52.6)$ \\
\hline$-80+$ & $250(26.9)$ & $678(73.1)$ \\
\hline - All age groups & $2787(46.7)$ & $3181(53.3)$ \\
\hline \multicolumn{3}{|l|}{ Sex } \\
\hline - Male & $1286(45.6)$ & $1535(54.4)$ \\
\hline - Female & $1501(47.7)$ & $1646(52.3)$ \\
\hline \multicolumn{3}{|l|}{ 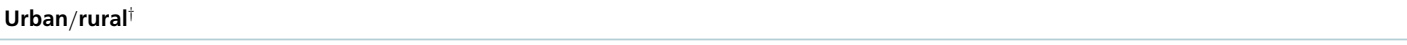 } \\
\hline - Urban & $2393(47.5)$ & $2647(52.5)$ \\
\hline - Rural & $152(42.1)$ & 209 (57.9) \\
\hline - Rural-remote & $165(41.5)$ & $233(58.5)$ \\
\hline - Rural-very remote & $75(46.9)$ & $85(53.1)$ \\
\hline \multicolumn{3}{|l|}{ Immigration tercile $^{\dagger}$} \\
\hline - 1 (Lowest) & $1781(44.9)$ & $2184(55.1)$ \\
\hline-2 (Medium) & $604(48.7)$ & $636(51.3)$ \\
\hline - 3 (Highest) & $379(53.8)$ & $326(46.2)$ \\
\hline \multicolumn{3}{|l|}{ Income quintile ${ }^{\dagger}$} \\
\hline - 1 (Lowest) & $559(41.5)$ & $787(58.5)$ \\
\hline-2 & $595(47.1)$ & $667(52.9)$ \\
\hline-3 (Middle) & $569(48.5)$ & $605(51.5)$ \\
\hline-4 & $555(49.4)$ & $568(50.6)$ \\
\hline - 5 (Highest) & $500(48.2)$ & $537(51.8)$ \\
\hline
\end{tabular}

Surgical resection rates \& equity measures

Gender and location of residence did not affect resection rates but patients with stage I and II NSCLC (Cohort $\mathrm{A}+\mathrm{B})$ in the highest income quintiles underwent surgical resection more commonly $(56.0 \%)$ than those in the lowest income quintile $(50.8 \% ; \mathrm{p}=0.04)$. Patients from those areas of Ontario with the highest numbers of immigrants also had higher resection rates compared with those with the lowest numbers of immigrants (60.4 vs $53.4 \% ; \mathrm{p}=0.003)$.

\section{Concordance with AC guideline}

A total of 332 of 616 of Cohort A patients (53.8\%, CI: 49.9-57.8\%) with stage II/IIIA NSCLC received guideline concordant AC (Table 2). The rate was modestly higher in cohort B at 56.1\% (CI: 52.2-59.8\%) but was not statistically significant. The age-adjusted percentage of resected stage II or IIIA NSCLC receiving AC by health region ranged from a low of $34.6 \%$ to a high of $71.4 \%$ in Cohort $\mathrm{A}$ and from 43.2 to $84.6 \%$ in Cohort $\mathrm{B}$ but was statistically different from the provincial average in only one health region. Most LHINs showed a small increase in the proportion of patients undergoing postoperative AC but there were also four LHINs, where a small but statistically insignificant decrease in the percentage of patients receiving AC was noted. One LHIN [\#11] had a substantial drop from 71.4 to $49.1 \%$; $\mathrm{p}=0.02$ ).

The guideline concordance by age is shown in Table 3. In Cohorts A and B and for both cohorts combined, patients over the age of 65 years were significantly less likely to be treated with $\mathrm{AC}$ as compared with younger patients ( $\mathrm{p}<0.0001$ ). More female patients received AC in Cohort A (females 58.0\%, CI: 52.4-63.3\%) compared with males (49.7\%, CI: 43.9-55.1\%; $\mathrm{p}=0.04)$, although this difference was not apparent in Cohort B. Patients from rural areas were generally treated with $\mathrm{AC}$ as frequently as urban dwellers except if they lived in very remote locations $[65.0 \%$ of rural patients in Cohort B received AC (CI: 49-78.5\%) compared with 55.4\% (CI: 51.1- 
Table 2. Percent concordance with adjuvant postoperative chemotherapy guideline by cohort and region.

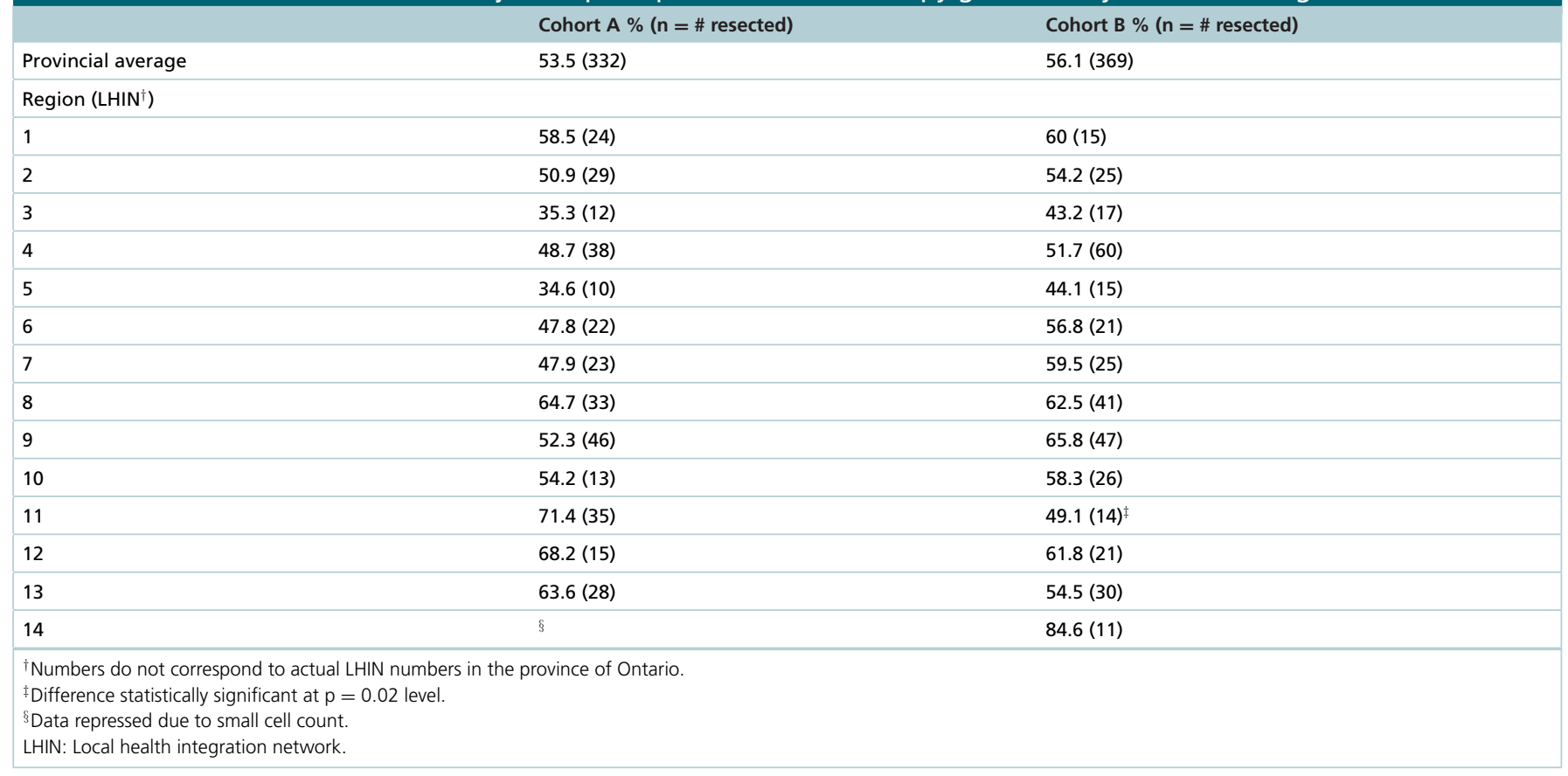

\section{Table 3. Frequency of adjuvant chemotherapy administration in stage II and IIIA non-small-cell lung cancer by equity} measure.

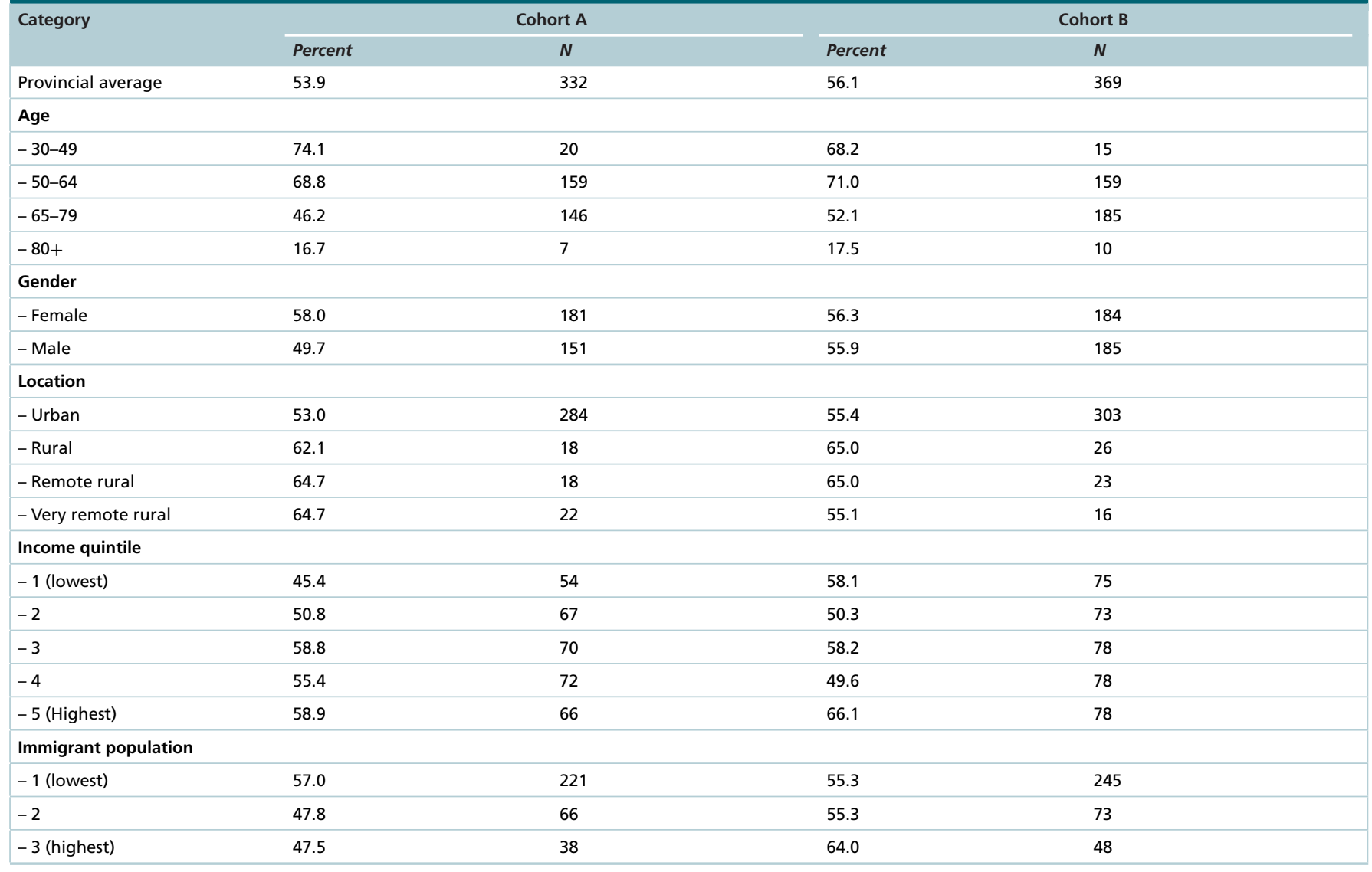


59.5\%) of those living in urban settings]. A significantly greater percentage of patients from high income quintiles (58.9\%) received AC compared with $45.4 \%$ in the lowest quintile in Cohort A. A similar pattern was evident in Cohort B. An inconsistent pattern was seen with regard to immigrant population, possibly due to the small number of cases in the terciles with the highest immigrant populations.

\section{Discussion}

The Cancer Quality Council of Ontario selected the guideline on AC for resected NSCLC to monitor concordance because of the evidence of a survival benefit and the fact that the guideline recommendation represented a relatively recent change in practice. It was anticipated that the concordance rate with the AC guideline would be high, which would signal that Ontario lung cancer patients were receiving current best practice. It was also anticipated that the adoption rate of AC would increase over the time due to promotion of best practice through CCO's Lung Disease Pathway Management initiative [25]. Therefore, it was surprising to see how low the rates of concordance were in both time periods for $\mathrm{AC}$ and the degree of regional variance in guideline concordant care.

Although it is commonly assumed that virtually all lung cancer patients receive current best practice as articulated in current practice guidelines, this is clearly not the case at a population level even within a publicly funded health care system. The reasons for this are uncertain but likely include the fact that the patients selected for entry into clinical trials are not representative of the wider lung cancer population as they tend to be younger and to have few comorbidities. The median age of patients in clinical trials is typically 60 years, whereas Canadian Cancer Registry statistics show that $53 \%$ of lung cancer patients are older than 70 years and $4.6 \%$ are 80 years or over [26]. During CCO's Lung Cancer Disease Pathway initiative, it was noted that $29 \%$ of NSCLC patients diagnosed in the province of Ontario in 2011 received no active treatment at all. $69 \%$ of these patients were older than 70 years and $32 \%$ were over 80 years. The lack of data on the comorbidities of the patients included in our study limits the interpretation of the data; however, it is reasonable to assume that many of these elderly patients did not receive treatment because of frailty, comorbidities or patient choice. Nonetheless, $10 \%$ were 60 years of age or younger and the explanation for not receiving $\mathrm{AC}$ is unclear.

It was also noteworthy and somewhat surprising that a significant number of stage I and II patients received no surgical treatment. Relatively few older patients ( $>80$ years) underwent curative surgical resection, which is perhaps not unexpected. However, only 55\% of younger patients (50-64 years) had a resection. Despite Ontario's publically funded health care system, patients from the lowest income populations were less likely to have a surgical resection. The reasons for this disparity are unclear but ability to access services, health literacy and comorbidities may be factors. Variability in surgical resection rates has also been noted in other population-based studies notably in England where resection rates for NSCLC ranged from 3 to 18\% according to geographic region [27].

With regard to the use of $\mathrm{AC}$, the results seen in this population-based study are consistent with a number of institutional and small jurisdictional studies. These studies provide insights into why patients do not receive AC. A retrospective chart review of 204 patients with completely resected stages I-IIIA NSCLC treated between May 2003 and May 2005 at the Toronto General Hospital included 59 patients with stage IA disease, 77 with IB, 8 with IIA, 41 with IIB and 19 with stage IIIA [28]. After the results of the BR.10 and Cancer and Leukemia Group B 9633 were reported in June 2004, 63\% (56/89) of surgically resected patients were referred for AC. The reasons for not referring to a medical oncologist included stage IA disease, the surgeon's opinion that adjuvant therapy was inappropriate, the patient declined, comorbidities, postoperative complications and advanced age. Of 92 patients referred to medical oncology, only $42(46 \%)$ received AC. The reasons for not prescribing AC included patient refusal (50\%), comorbidities (14\%), stage IA disease (10\%) and advanced age (4\%).

A retrospective study of all patients with NSCLC who underwent curative-intent surgery in Nova Scotia, Canada in 2005 also showed a relatively low rate of use of AC [29]. Of the 108 patients, referral to medical oncology occurred in only $44 \%$. Thirty of these 41 patients (73\%) had stage II-III disease and of these, $22(54 \%)$ received AC. Higher uptake was significantly associated with younger age, disease stage (II/III vs I) and surgery type (pneumonectomy vs wedge resection).

Tognela et al. reported a similar result from the South Western Sydney Local Health District of Australia) [30]. Of 137 patients who underwent surgical resection, only 63 (46\%) received AC. The main reasons for patients not receiving AC included stage IB disease (32\%), advanced age/comorbidities (24\%), patient preference (14\%), prior neoadjuvant treatment $(7 \%)$ and nonreferral $(7 \%)$.

Cuffe et al. previously explored the relationship between age and the frequency of administration of AC using Ontario Cancer Registry data [31]. From 2001 to 2006, 6304 patients with NSCLC underwent surgical resection 
and $43.8 \%$ of these patients were elderly (age $\geq 70$ years). The uptake of AC was low although it increased from $3.3 \%$ in the period $2001-2003$ to $16.2 \%$ in the period 2004-2006. The 4-year survival of elderly patients increased significantly over time ( $47.1 \%$ for patients diagnosed from 2001 to $2003 ; 49.9 \%$ for patients diagnosed from 2004 to 2006; $\mathrm{p}=.01$ ) and improved in all subgroups except for patients age $\geq 80$ years.

In the current study, it appears that more women than men accepted AC particularly in Cohort $\mathrm{A}(\mathrm{p}=0.04)$, although this difference was not significant in Cohort B. Income level appeared to make a difference with a greater proportion of patients receiving adjuvant therapy in the highest income level compared with the lowest for both cohorts. This is surprising in a publically funded health care system but may have more to do with factors such as comorbidities, health literacy and performance status. Neighborhoods with a higher immigrant population also appeared to receive AC less often. The explanation for this is unclear but could be because immigrant populations are less knowledgeable or accepting of treatment or that physicians are less able to communicate effectively the need for this adjuvant treatment to these populations. It is possible that a logistic regression analysis might help to clarify the reasons that underlie the greater use of adjuvant therapy in the higher income regions.

Our study is based on record linkage and has a number of limitations. Data on performance status, weight loss and comorbidities are not routinely captured in the administrative data sets that were mined for this evaluation and these characteristics are critical to clinical decision-making. It is now well recognized that these metrics need to be captured in order to evaluate whether patients are receiving quality cancer care. Patients completing the Edmonton Symptom Assessment Scale at electronic kiosks during visits to Ontario cancer centers are now also reporting their performance status.

Our data do suggest that greater efforts need to be made to understand the reasons behind the lower than expected level of compliance with treatments that can improve survival. A study of medical, radiation and surgical oncologists' knowledge of Ontario's practice guidelines and influence on treatment decisions found that physicians perceived their patients to be different from those in the clinical trials on which the practice guidelines were based [32]. Lung cancer patients were commonly older with significant comorbidities, which precluded use of AC. In addition, delays in referral and lack of administrative support were factors that contributed to non-treatment. This may indicate that there is a continuing negative attitude at the administrative level to the treatment of lung cancer patients.

Although it might be concluded that guideline adoption in Ontario has been relatively low and, therefore, not likely impactful on clinical outcomes, a study by Booth $e$ al. suggests the opposite [21]. Over the study period from 2001 to 2006, the proportion of patients receiving AC increased from 7\% (192 of 2950 patients) to 31\% (1032 of 3354 patients; $\mathrm{p}<.001$ ). Within 2 years of surgery, there was a $33 \%$ reduction in the proportion of patients admitted to hospital with metastatic disease $(\mathrm{p}<.001)$ and a substantial improvement in 4-year survival among surgically resected patients, from $52.5 \%(2001-2003)$ to $56.1 \%(2004-2006 ; p=0.001)$. The authors concluded that there was a rapid uptake of $\mathrm{AC}$ and that this was associated with a substantial improvement in overall survival. The failure to see much change between Cohorts A and B in the rate of uptake of AC was likely due to the fact that the most rapid increase in guideline uptake had already occurred prior to our study. Any further increase in the proportion of patients receiving postoperative $\mathrm{AC}$ will likely require efforts to address the equity issues we have identified.

Our data on concordance of lung cancer practice guideline recommendations with actual practice have demonstrated regional variance, which, in some cases, was significantly different from the provincial average. The reasons for these differences in practice are unclear but may be due to resource availability, physician attitudes to treatment or in the populations served.

These observations support the need for further research to understand the reasons behind the observed variation and the strategies that might reduce it.

Financial \& competing interests disclosure

The authors have no relevant affiliations or financial involvement with any organization or entity with a financial interest in or financial conflict with the subject matter or materials discussed in the manuscript. This includes employment, consultancies, honoraria, stock ownership or options, expert testimony, grants or patents received or pending, or royalties.

No writing assistance was utilized in the production of this manuscript. 


\section{Ethical conduct of research}

The authors state that they have obtained appropriate institutional review board approval or have followed the principles outlined in the Declaration of Helsinki for all human or animal experimental investigations. In addition, for investigations involving human subjects, informed consent has been obtained from the participants involved.

\section{Open Access}

This work is licensed under Crown copyright protection and licensed for use under the Open Government Licence unless otherwise indicated. Where any of the Crown copyright information in this work is republished or copied to others, the source of the material must be identified and the copyright status under the Open Government Licence acknowledged. Published under CC-BY 4.0 www.nationalarchives.gov.uk/doc/open-government-licence/version/3/ @ Crown Copyright

\section{References}

1. Cancer Care Ontario. www.cancercare.on.ca.

2. Program in Evidence-based Care. www.cancercare.on.ca/about/programs/pebc/

3. Lung Cancer Evidence-based Series (EBS) and Practice Guidelines (PG). www.cancercare.on.ca/toolbox/qualityguidelines/diseasesite/lung-ebs/

4. Anas R, Bell R, Brown A, Evans WK, Sawka C. A ten-year history: the Cancer Quality Council of Ontario. Healthcare Quarterly, Toronto, Ontario, 15 Spec No., 24-27 (2012).

5. Cancer System Quality Index. www.csqi.on.ca/

6. Committee on Quality of Health Care in America. Crossing the Quality Care. Crossing the quality chiasm: a new health system for the $21^{\text {st }}$ century. Institute of Medicine. National Academies Press, Washington DC, USA (2001).

7. Pisters KMW, Evans WK, Azzoli CG et al. CCO and the ASCO Guideline for adjuvant chemotherapy and adjuvant radiation therapy for stages I-IIIA resectable non-small cell lung cancer. J. Clin. Oncol. 25(34), 5506-5518 (2007).

8. Members of the Lung Cancer Disease Site Group. Postoperative Adjuvant chemotherapy, with or without radiotherapy, in completely resected non-small cell lung cancer. Ellis P, Agbassi C (Reviewers). Cancer Care Ontario, Toronto, Ontario, Jun 8, Program in Evidence-based Care Evidence-based Series No.: 7-1-2-Education and Information. (2012). www.cancercare.on.ca/common/pages/UserFile.aspx?fileId=14162

9 Bradbury P, Sivajohanathan D, Chan A et al. Postoperative Adjuvant Systemic Therapy in Completely Resected Non-Small Cell Lung Cancer. A Quality Initiative of the Program in Evidence-Based Care (PEBC), Cancer Care Ontario (CCO). 21 January 2016. www.cancercare.on.ca/common/pages/UserFile.aspx?fileId=351861

10. Winton T, Livingston R, Johnson D et al. Vinorelbine plus cisplatin vs. observation in resected non-small-cell lung cancer. N. Engl. J. Med. 352(25), 2589-2597 (2005).

11. Le Chevalier T, Brisgand D, Douillard JY et al. Randomized study of vinorelbine and cisplatin versus vindesine and cisplatin versus vinorelbine alone in advanced non-small-cell lung cancer: results of a European multicenter trial including 612 patients. J. Clin. Oncol. 12, 360-367 (1994).

12. Arriagada R, Bergman B, Dunant A et al. Cisplatin-based adjuvant chemotherapy in patients with completely resected non-small cell lung cancer. N. Engl. J. Med. 350, 351-360 (2004).

13. Dunant A, Pignon J, Le Chevalier T. Adjuvant chemotherapy for non-small cell lung cancer: contribution of the International Adjuvant Lung Trial. Clin. Cancer Res. 11(Suppl. 13), 5017s-5021s (2005).

14. Douillard JY, Rosell R, De Lena M et al. Impact of post-operative radiation therapy on survival in patients with complete resection and stage I, II, IIIA non-small-cell lung cancer treated with adjuvant chemotherapy: the Adjuvant Navelbine International Trialist Association (ANITA) randomized trial. Int. J. Radiat. Oncol. Biol. Phys. 72, 695-701 (2008).

15. Douillard J-Y, Tribodet H, Aubert D et al. Adjuvant cisplatin and vinorelbine for completely resected non-small cell lung cancer: Subgroup analysis of the Lung Adjuvant Cisplatin Evaluation. J. Thorac. Oncol. 5(2), 220-228 (2010).

16. Strauss GM, Herndon JE, Maddaus MA et al. Adjuvant paclitaxel plus carboplatin compared with observation in stage IB non-small-cell lung cancer: CALGB 9633 with the Cancer and Leukemia Group B, Radiation Therapy Oncology Group, and North Central Cancer Treatment Group Study Groups. J. Clin. Oncol. 26, 5043-5051 (2008).

17. Non-Small-Cell Lung Cancer Collaborative Group. Chemotherapy in non-small-cell lung cancer: a meta-analysis using updated data on individual patients from 52 randomised clinical trials. BMJ 311, 899-910 (1995).

18. Pignon JP, Tribotet H, Scagliotti GV et al. Lung Adjuvant Cisplatin Evaluation (LACE): a pooled analysis by LACE Collaborative Group. J. Clin. Oncol. 26, 3552-3559 (2008).

19. Butts CA, Ding K, Seymour L et al. Randomized Phase III trial of vinorelbine plus cisplatin compared with observation in completely resected stage IB and II non-small-cell lung cancer: Updated survival analysis of JBR-10. J. Clin. Oncol. 28, 29-34 (2009). 
20. Ng R, Hasan B, Mittmann N et al. Economic analysis of NCIC CTG JBR.10: a randomized trial of adjuvant vinorelbine plus cisplatin compared with observation in early stage non-small-cell lung cancer-a report of the Working Group on Economic Analysis, and the Lung Disease Site Group, National Cancer Institute of Canada Clinical Trials Group. J. Clin. Oncol. 25(16), 2256-2261 (2007).

21. Booth CM, Shepherd FA, Peng Y et al. Adoption of adjuvant chemotherapy for non-small-cell lung cancer: a population-based outcomes study. J. Clin. Oncol. 28(21), 3472-3478 (2010).

22. International Classification of Diseases for Oncology (ICD-O) - 3rd edition, 1st revision. World Health Organization, Geneva, Switzerland (2013).

23 Collaborative staging (2017). https://seer.cancer.gov/tools/collabstaging/index.html

24. Data and definitions - Statistics Canada (2017). www.statcan.gc.ca/pub/21--006-x/2008008/section/s2-eng.htm

25. Evans WK, Ung YC, Assouad N, Chyjek A, Sawka C. Improving the quality of lung cancer care in Ontario: the lung cancer disease pathway initiative. J. Thorac. Oncol. 8(7), 876-882 (2013).

26. Canadian Cancer Society. Advisory Committee on Cancer Statistics. Canadian Cancer Statistics 2013. Canadian Cancer Society, Toronto, Ontario (2013).

27. Riaz SP, Luchtenborg M, Jack RH et al. Variation in surgical resection for lung cancer in relation to survival: population-based study in England 2004-2006. Eur. J. Cancer 48(1), 54-60 (2012).

28. Kassam F, Shepherd FA, Johnston $\mathrm{M}$ et al. Referral patterns for adjuvant chemotherapy patients with completely resected non-small cell lung cancer. J. Thorac. Oncol. 2(1), 39-43 (2007).

29. Younis T, Al-Fayea T, Virik K, Morzycki W, Saint-Jacques N. Adjuvant chemotherapy uptake in non-small cell lung cancer. J. Thorac. Oncol. 3(11), 1272-1278 (2008).

30. Tognela A, Lim S, Descallar J et al. Patterns of care in patients receiving adjuvant chemotherapy for resected non-small cell lung cancer (NSCLC) in South Western Sydney Local Health District (SWSLHD) World Conference on Lung Cancer. 27-30 October 2013 (Abstract P3 12-009 15th).

31. Cuffe E, Booth CM, Peng Y et al. Adjuvant chemotherapy for non-small cell lung cancer in the elderly: a population based study in Ontario, Canada. J. Clin. Oncol. 30(15), 1813-1821 (2012).

32. Brouwers MC, Makarski J, Garcia K et al. A mixed methods approach to understand variation in lung cancer practice and the role of guidelines. Implement. Sci. 9(1), 36 (2014). 